\title{
Partner Modeling Is Mutual
}

\author{
Mirweis Sangin, Nicolas Nova, Gaëlle Molinari, Pierre Dillenbourg, \\ Ecole Polytechnique Fédérale de Lausanne (EPFL), School of Computer and Communication Sciences, CRAFT, \\ EPFL-CRAFT CE 1631 Station 1 CH-1015 Lausanne Switzerland, \\ \{mirweis.sangin, nicolas.nova, gaelle.molinari, pierre.dillenbourg\}@epfl.ch
}

\begin{abstract}
Collaborative learning has been hypothesized to be related to the cognitive effort engaged by co-learners to build a shared understanding. The process of constructing this shared understanding requires each team member to build some kind of representation of the behavior, beliefs, knowledge or intentions of other group members. This contribution reports interesting findings regarding to the process of modeling each other. In two empirical studies, we measured the accuracy of the mutual model, i.e. the difference between what A believes B knows, has done or intends to do and what B actually knows, has done or intends to do. In both studies, we found a significant correlation between the accuracy of A's model of B and the accuracy of B's model of A. This leads us to think that the process of modeling one's partners does not simply reflect individual attitudes or skills but emerges as a property of group interactions. We describe on-going studies that explore these preliminary results.
\end{abstract}

\section{Introduction}

It is now broadly admitted that learners do not benefit from collaboration simply because they are in a group but because collaboration triggers additional activities such as explanation, disagreement and mutual regulation (Dillenbourg, 1999). After Roschelle and Teasley (1995), many CSCL scholars conceptualized collaborative learning as an activity in which shared knowledge is constructed by peers through their interactions with each other and also with their environment. The notion of shared knowledge is derived from psycholinguistic concept of "grounding" (Clark \&Wilkes-Gibbs, 1986): during interactions, the "interactants" constantly try to ensure of a good mutual understanding. Grounding is the collective process by which the individuals engaged in a conversation try to guarantee their mutual understanding. 'Shared understanding' or 'mutual understanding' are very intuitive concepts, both for analyzing interactions and for designing applications, which probably explains their impact on CSCL. However, this notion is questioned both within psycholinguistics (Sperber \& Wilson, 1986) and within CSCL (Baker, Hansen, Joiner \& Traum, 1999; Koschmann \& LeBaron 2003). Our research questioned it in a different way: we zoomed in on the mechanics of grounding, by analysing how a shared solution emerges from the sum of a long sequence of contributions (Dillenbourg \& Traum, 2006). Our approach goes one step further: We attempt to understand the socio-cognitive benefits of co-constructing a shared understanding. We investigate a mechanism that is hypothesized to lie at the heart of grounding. For Clark and Brennan (1991), common grounds are a set of mutual beliefs defined as the amount of information shared (e.g. presuppositions, knowledge, assumptions, beliefs). Establishing this set of beliefs requires that the co-learners build some representation of their partners' knowledge, beliefs and goals. We referred to the process of building assumptions about the beliefs and the knowledge of their partner(s) as mutual modeling (Dillenbourg, 1999). However the abstract and unobservable aspect of this process raises methodological challenges regarding its apprehension by researchers. Therefore, this contribution attempts to ask the general question of the socio-cognitive nature of mutual modeling, by assessing whether the process of modeling one's partner is grounded at the individual or the group level.

The paper does not describe environments for collaborative learning but reports basic research on the sociocognitive mechanisms related to mutual modeling. The first section explores the concept of mutual modeling and its relationship with CSCL features such as scripts and awareness tools. The second and third sections report empirical studies in which the accuracy of mutual models has been measured. The conclusion section describes how the hypotheses that come out of these two studies are currently being investigated through two other studies, which focus on CSCL setting with a stronger educational flavor.

\section{Mutual modeling in collaborative task}

The ability to understand the partner's understanding and to adapt to his/her viewpoint has been investigated under the labels of intersubjectivity (Wertsch, 1985; Bromme, 2000) and audience design (Lockridge \& Brennan, 2002). Suthers (2006) recently proposed "the technology affordances of intersubjective meaning-making" 
as an integrative agenda for CSCL research. In his sense, a common denominator of understanding learning in a collaborative setting is the peers' attempt to make sense of situations and of each other. Intersubjectivity is played in the field of the physical and historical context available to the participants to jointly compose interpretations, which could be considered as a new gist - alternative to the notion of producing mutual-beliefs about making unshared information shared - for collaborative learning. To sum up, taking into account the peer's perspective is a crux facet of intersubjectivity on which most of the social activities rely. As highlighted by Malle (2003), the ability to represent and reason about self and other's mental states (e.g. beliefs, desires, intentions, mere thoughts, experiences, emotions, attitudes) is a great achievement of the human mind evolution that is considered as a prerequisite for many social and cognitive processes such as natural language acquisition, social interaction, reflexive thought, and moral development.

The term "mutual modeling" does not imply that collaborators maintain a detailed representation of their partner's knowledge, nor an explicit one. Simply stated, if A wants to (dis-)agree with B, A needs some representation of B's intentions; if A wants to repair B's misunderstanding, A needs some representation of what B has understood. Mutual modeling is as functional as the grounding process: the degree of accuracy depends on the task requirements; it has to be extremely high if two pilots collaborate on landing a plane, as in Hutchins' (1995) observations, but can be much lower if they discuss about their last party (down to what politeness allows). Moreover, this mutual model is not constructed in a vacuum but is based on multiple inference mechanisms. Common grounds are initialized by the assumptions people make about their partner from cues such as his/her community membership (age, culture, profession, ...) and from co-presence (e.g. common ground includes any event to which A and B attended together) (Clark \& Marshall, 1981). Several scholars studied how this initial model imparts on communication, namely because it can easily be manipulated. For instance, Slugoski, Lalljee, Lamb \& Ginsburg (1993) pretended to the subjects that their (fake) partner has or not received the same information. They observed that the subjects adapt to their partner by focusing the explanation on the items that (s)he is supposed to ignore. Brennan (1991) showed that the subjects used different initial strategies in forming queries depending on who they were told their partner was. Other simpler inference mechanisms such as default reasoning rules (e.g. B agrees with me unless he disagrees) are developed according to the conversational context. The mutual modeling could not occur independently from culturally acquired interaction schemata that constrain the space of interpretation of the other's behavior. Actually, the CSCL notion of 'scripts' (Dillenbourg, 2002) can be conceptualized as providing co-learners with an explicit schema that narrows down the space of interpretations and hence serves as prosthesis for mutual modeling. Another prosthesis for mutual modeling is the notion of awareness tools (Greenberg, Roseman, 1996); these are features of CSCW environment in which A is informed about B's actions that A does not directly perceive.

Even when mutual modeling is not detailed and explicit, reasoning on what one's partner believes involves some cognitive load. For Clark \& Wilkes-Gibbs (1986), what is important is not the individual effort made by the receiver of a communicative act, but the overall least collaborative effort. The cost of producing a perfect utterance may be higher than the cost of repairing the problems which arise. For instance, subjects are less careful about adapting utterances to their partner when they know they can provide feedback on his/her understanding (Schober, 1993). We introduced instead the notion of 'optimal collaborative effort' (Dillenbourg et al, 1996) to stress the fact that misunderstanding should not be viewed as something to be avoided (even if this were possible), but as an opportunity to explain, to justify, and so forth. Here we enter into the global argument regarding cognitive load in learning activities, namely in discovery learning environments: there is no learning without cognitive load, but overload may hinder learning (Paas, Renkl \& Sweller, 2003). In the context of collaborative learning, we understand the cognitive load induced by mutual modeling as part of Schwartz (1995) notion of effort towards a shared understanding. For instance, conflict-resolution scripts or JIGSAW scripts are purposely designed for augmenting (reasonably) the effort group members have to engage to reach a shared solution.

Mutual modeling has many dimensions, from which we dissociated 'dispositional' versus 'situational' aspects. The 'dispositional' aspects refer to A's representation of B's long term knowledge, skills or traits. It is hence closely related to the notion of transactive memory (Wegner, 1987; Moreland, 2000). 'Situational' aspects refer to A's representation of B's knowledge, behavior or intention specifically activated in the situation in which A and B collaborate.

This leads us to the long term research question that underlies our work: does the mutual modeling process contribute to the learning outcomes of collaborative problem solving? This question is difficult to investigate 
because the degree of mutual modeling is both difficult to manipulate as an independent variable, and difficult to measure as a dependent variable. Measuring it is difficult because, as soon as one asks learners what their partner knows, is doing or intends to do, we trigger a modeling process beyond what it would 'naturally' be. Controlling the degree of mutual modeling is also difficult. As we mentioned earlier, scripts and awareness tool potentially influence the mutual modeling process, being some kind of prosthesis. Now, as for any prosthesis involved in learning, we ignore whether scripts and awareness tools will augment the mutual modeling process (by scaffolding it) or inhibit it (by making it useless). Moreover, it is difficult to estimate the accuracy of a mutual model in absolute terms. Hence, this contribution focuses on a simple question: considering $M(A, B)$ as being $A$ 's representation of $B$, what is the relationship between $M(A, B)$ and $M(B, A)$ ?

Actually, an alternative hypothesis is that participants do not build a representation of their partners' mental states but instead a representation of the interaction process at the group level: instead of modeling who knows what, who does what or who said what, the team members could maintain a representation of what the team knows, has done or has said. We refer to this as the group model. This alternative is directly inspired by distributed cognition theories (Pea, 1993; Salomon, 1993; Hutchins 1995) and the team mental model (Canon-Bowers, Salas, Converse, 1993). The two hypotheses are of course complementary since these two models feed each other.

This contribution does not answer these general research questions but report some results collected in two empirical studies on mutual modeling, respectively in a virtual environment and in real space. These results are discussed under the light of social and cognitive theories. The discussion also mentions on-going studies on the mutual modeling process within more traditional collaborative learning settings.

\section{Study 1.}

We attempted to measure mutual modeling by using awareness tools in a collaborative video game called Spaceminers. The research question was to study the impact of an awareness tool on group performance and mutual modeling. The availability of an awareness tool was our independent variable. The main results have been published in (Nova, Wehrle, Goslin, Bourquin, Dillenbourg, 2006). We focus here on the question addressed in the introduction, that is the relation between the modeling performed by each user or the relation between $M(A, B)$ and $\mathrm{M}(\mathrm{B}, \mathrm{A})$. Our main dependent variable is the mutual modeling accuracy, hereafter referred to as MM-accuracy

\section{Experiment design}

SpaceMiners is a 3D computer game that involves two players in space missions in which they have to collect minerals located in asteroids and bring them to a space station. To do so, they shoot drones through the space after choosing their initial direction and speed. Once launched, the trajectory of drones is only influenced by the gravity of planets and by specific tools that players collaboratively position between planets.

During the experiment, the teams were confronted with three increasingly complex situations. The experiment was 2 hours long, with a 30 minutes tutorial and 3 levels of 30 minutes. Thirty-six persons participated in this study, all native French speakers. We constituted 18 pairs of participants $(\mathrm{N}=18)$ who were not familiar with each other. The pairs were assigned randomly to either the Control condition (without the awareness tool) or the awareness condition (with the awareness tool). In the awareness condition, team members could view what their partner was looking at and hence were expected to infer more accurately his/her teammate's intentions. Each player sat in front of a distinct computer located in different rooms. They interacted with the game using a regular Logitech joystick and communicated with each other through an audio channel.

\section{Measures}

Task performance was measured as the score reached by the subjects after three situations. In order to evaluate the mutual modeling accuracy during the task, we used two questionnaires as shown on Figure 1. Both of them were displayed during each of the 3 phases of the game, as a transparent layer appearing on the game level. The first questionnaire concerned the player's intended actions. It asked each player about what they were intending to do at the moment (guiding his partner, trying to understand his strategy, trying to establish a common strategy, adjusting a shoot, etc.). Then, the second questioned asked each player about what he thought the partner was intending to do. Some answers were identical in both questionnaires (like "adjusting a shoot") while others were reversed. For instance, the answer "guide him" will be reversed as "guide me" and vice-versa. Each questionnaire then had 10 questions that covered the basic actions that could be performed. 


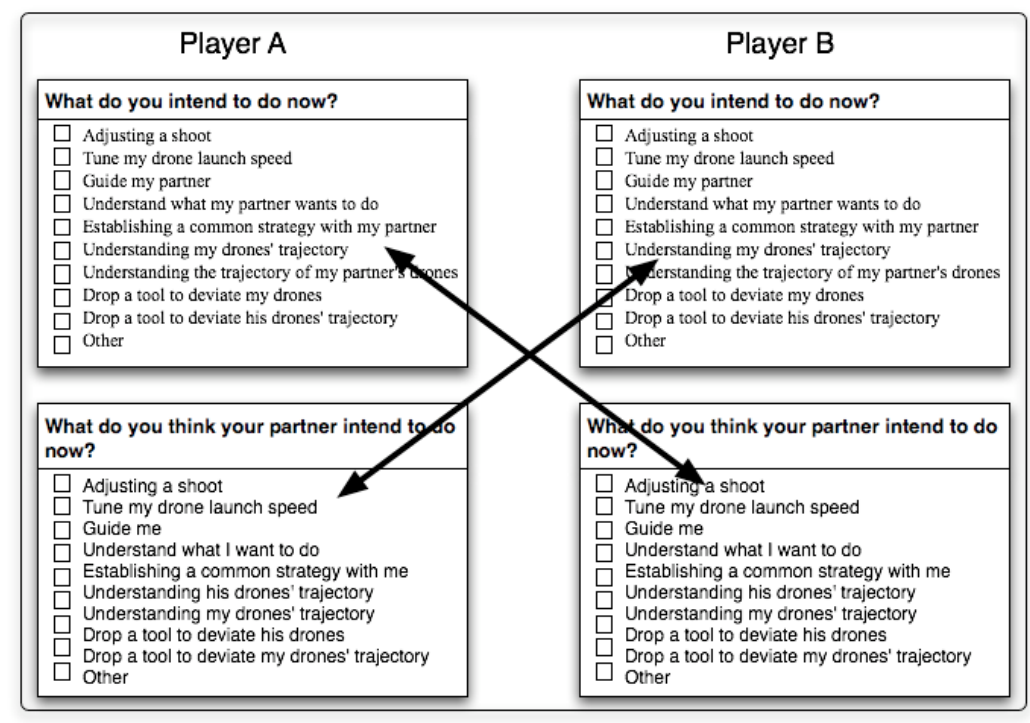

Figure 1: Crossed questions for measuring mutual modeling accuracy.

These questionnaires gave us the possibility to compare player A's prediction about B's intentions with B's self-declared intentions. Of course, this method faces the limits of any questionnaire in which somebody has to selfdeclare his or her intentions. We compared the first answer of a player (about what $\mathrm{A}$ is intending to do) to the answer of his partner to the second question (about what B believes A is doing). Our estimation of MM -accuracy has been computed as the number of common answers given by the two players to those two questionnaires: does A's prediction of B's answer matches B's actual answer? Since there were 3 evaluations (one per level), we computed the MM-accuracy per individual for each level of the game. The global MM-accuracy is the sum of these 3.

\section{Findings and Discussion}

The awareness tool permitted higher group performance, but it did not improve the accuracy of the mutual model. However, within the experimental group, the pairs who intensively used the awareness tools obtained a significantly higher MM accuracy (for more details, see Nova et al., 2006). In order to compare M (A,B) and M (B,A), we computed intraclass correlation as described by Kenny et al. (1998) from the answers to the crossquestionnaires. We found a positive and significant correlation $(r=.38, p<.05)$ between $M(A, B)$ and M (B.A). This sounds as a minor result for this particular study but actually conveys an important outcome: mutual modeling appears to be a group variable rather than a personal activity. We expected MM-accuracy to be a personal parameter, i.e. that some participants spontaneously pay more attention or engage more effort in monitoring their peer. This could be due to some social attitude or to specific cognitive skills required to build a mutual model. This strong correlation supports a different hypothesis in which mutual modeling emerges as a property of the quality of interactions among peers: some pairs seems to collaborate in such a way that their verbal and non-verbal interactions produce more cues available to both partners so that they can build a mutual model. This does not remove individual variability (correlation was not 1 ). Interestingly, we found that the relation between $M(A, B)$ and $M(B, A)$ was not very different in the two conditions: the average absolute difference between MM-Accuracy $(A, B)$ and MMaccuracy $(\mathrm{B}, \mathrm{A})$ is not significantly different with or without the awareness tool $(\mathrm{F}[1,13]=0.1445$, $\mathrm{p}$-value $=$ 0.7097)

\section{Study 2}

In this second study, instead of evaluating mutual modeling during the task, we chose to measure it after task completion. This experiment was based on a pervasive game called Catchbob. As for the previous experiment, this game was used to evaluate the influence of awareness tools on group performance and MM-accuracy, but we will focus here on the results about the relationship between $M(A, B)$ and $M(B, A)$.

\section{Experimental design}


Catchbob is an experimental platform implemented as a mobile game in which groups of 3 players have to solve a joint task. The game was played on the school campus and participants had to find a virtual object ('Bob') and catch it by forming a triangle around it. Players used a Tablet PC that displays a map of the campus and an indication of their personal distance from Bob. Their annotations on the map were shared with the two other players, but fadeout after a few minutes. The awareness tool displayed the location of the two other players on the map. Hereafter, we refer to this information as mutual location awareness (MLA). It constituted our independent variable.

In this study, we selected groups of students from the same class and hence who knew each other. Ninety students participated in this experiment. We assigned 10 groups of 3 persons to each of our three experimental conditions: the control condition (without MLA) and two experimental conditions: synchronous MLA (display current position of each player) and asynchronous MLA (display current position of each player and their spatial trace). We controlled group gender so that each condition was made up of $25 \%$ of female and $75 \%$ of male.

\section{Measures}

As dependent variable, we measured MM-accuracy by asking players to draw their own path and the one of each of their partners after the game. This enabled us to calculate the number of errors players made while drawing the path of their partners. We compared the path player A attributed to B with B's real paths recorded by the system and the same for $\mathrm{A} \& \mathrm{C}$ or $\mathrm{B} \& \mathrm{C}$ as depicted on Figure 2.
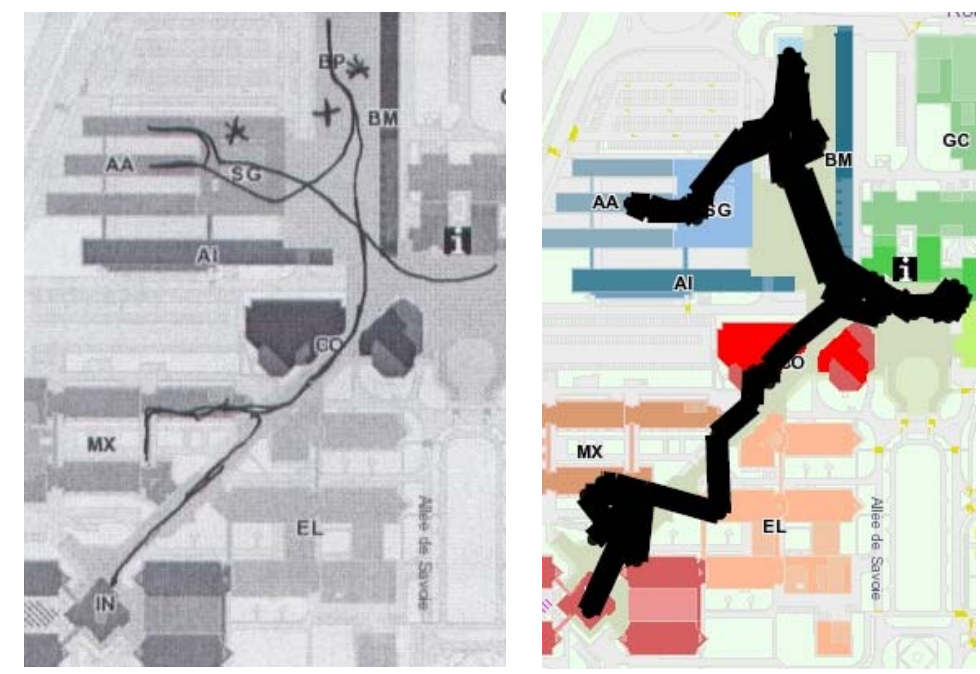

Figure 2: (Left) Drawing A made of B's path; (Right) Real path followed by B as extracted from the logfile.

We computed the number of errors between $\mathrm{M}(\mathrm{A}, \mathrm{B})$ and $\mathrm{M}($ system, B). What we counted as an error where either a place where the partner have not been or a place where he/she had gone but he/she has not drawn. Three criteria have been defined to describe what an error was: distance (if the line was longer than the maximum size of our campus corridor), presence of an obstacle (door/wall/glass), walking back was not perceived as an error. An individual MM-accuracy is the sum of errors made by a player about his/her two partners' paths. We calculated MM-accuracy for each individual (M (A,B), M (B,A), M (A, C), M (B,C),...) and for each group (the sum of the individual measures). It is important to stress that subjects made very few mistakes when drawing their own path on the campus ( $85 \%$ made 0 errors). This enables us to consider mistakes in their partners' path as being due to a lack of mutual modeling accuracy instead as being due to spatial skills (e.g. a difficulty in reporting trajectories on a map).

\section{Findings and Discussion}

We did not find any significant difference regarding the task performance between the three experimental conditions. However, our surprise was that the absence of the awareness tool led player to higher MM-accuracy: players better remember their partners' path if they had not seen their position permanently. We will not enter into the details of these results but simply stress that teams without MLA did more annotations on the map. It seems that permanent MLA has an underwhelming effect (Nova et al., 2005). Let us now focus on the relationship between M 
$(\mathrm{A}, \mathrm{B})$ and $\mathrm{M}(\mathrm{B}, \mathrm{A})$. We checked the intra-group dependence of the results through the computation of intra-class correlation: the correlation is again positive $(\mathrm{r}=.41)$ and significant $(\mathrm{p}=.01)$. The number of errors made by the subjects is correlated with the number of errors made by the other partners. This result confirms the correlation found in the first study. This second result is even more surprising for us than the former: despite the high heterogeneity of spatial representation skills among adults (see for instance Liben et al, 1981), this high correlation indicates again that MM-accuracy reflect more group processes than personal features. Since team members did not interact massively during the task, the intra-group correlation may not be explained by the quality of verbal interactions but by other aspects of their collaboration, probably the quality of the task strategy that emerged in the group. However, the relation between strategy and MM-accuracy is complex: if we do a post-hoc split, groups with a high level of MM-accuracy do not perform better than pairs with low MM-accuracy $(F[1,17]=1.4456, p=$ $0.2452)$.

\section{Discussion and further studies}

These two studies results revealed a correlation between the model peers build about each others' behaviors and intentions. Simply stated, if a team member A build an accurate model of his or her partner B, then B also tends to build an accurate model of $\mathrm{A}$. The conclusion we draw at this point is that the activity of modeling the partner is not reciprocal but mutual. A reciprocal relationship means that modeling is mainly an individual activity where $A$ infers $M(A, B)$ from B's actions and utterances. A mutual relationship implies that $M(A, B)$ and $M(B, A)$ are jointly constructed through interactions. The term 'mutual' may mean that not only A builds $M(A, B)$, but he also builds $\mathrm{M}(\mathrm{A}, \mathrm{M}(\mathrm{B}, \mathrm{A}))$. We will not enter in the long debate on the possibility of an infinite regress of nested models (discussed in Smith, 1982 or in Clark, 1996). Another interpretation is that team members actually build a model of the group-in-interaction, something like $\mathrm{M}(\mathrm{A}, \mathrm{AB})$. We are not able to choose among different hypotheses at this stage, since the reported experiments were not designed for exploring these issues.

These findings emerged as side-effects of other questions. The positive aspect of these results is that the correlation has been observed in two different contexts: virtual space in study 1 versus real space in study 2, groups of 2 in study 1 versus groups of 3 in study 2. Moreover, these results have been found using different methods: on-task in study 1 versus off-task in study 2, subjective validation (comparing A's model to B's answer) in study 1 versus objective validation in study 2 (comparing A's model with B's behavior). This diversity somewhat consolidates our results but these results are still preliminary: the selected tasks were not proper learning tasks and, overall, we still face serious methodological difficulties. On the one hand, asking learners 'on task' what their partner knows, is doing or intends to do triggers a modeling process which could alter the natural modeling process, and on the other hand, providing learners with an 'after-task' survey may imply mnemonic and rationalization biases. In other words, the abstract and unobservable characteristics of the mutual modeling process imply methodological challenges that call for indirect measures and assessment methods. Furthermore, mutual modeling in everyday life involves a large variety of mental states to be represented such as knowledge, behaviors, beliefs, desires, intentions, emotions, traits, attitudes, etc. Three of these mental states are particularly relevant in collaborative learning situations, namely inferences about partners' knowledge, behavior, goals (intentions). Study 1 focused essentially on inferences about peers' intentions while study 2 investigated inferences about peers' behavior. Our on-going study focuses on the inferences about peers' knowledge that is expected to be the most important aspect of mutual modeling in collaborative learning.

Our current empirical studies investigate the mutual modeling process in conceptual learning. The goal of these experiments is twofold. Our theoretical question is whether or not the mutual modeling effort enhances collaborative learning gains. Our methodological question is to capture the mutual modeling mechanisms. In order to avoid the 'anticipation' and 'rationalization' biases, we use interaction analyses and parallel gaze analysis. We are therefore using two eyes-tracking machines and we perform an automatic comparison of the eye paths of both learners as in (Richardson \& Dale, 2005). These experiments use the two MM prostheses described in the introduction, awareness tools and scripts. In both cases, subject start by reading a text individually (Phase 1) and then have to build a concept map together (Phase 2). In the first experiment, the independent variable is an awareness tool available during Phase 2: A is informed of B's knowledge on three different chapters of the learning material; this knowledge has been previously measured through a pre-test at the end of Phase 1 . In the second experiment, different scripts are implemented by providing subjects with complementary partial texts (jigsaw script) or conflictual texts (argumentation scripts) in phase 1. Both of these experiments manipulate the mutual modeling process in complex collaborative learning situations. Awareness tools about peer's knowledge (and behavior in general) may trigger mutual modeling facilities whereas the 'collaborative scripts' may strain effort of mutual understanding and by 
extension, enhance mutual modeling and perspective taking and making efforts. In a circular (if not spiral) manner, this increase in the mutual modeling effort may elicit interaction processes such as audience design, mutual regulation, elaborated explanation asking and providing, which are known to be beneficial for learning.

\section{References}

Baker, M., Hansen, T., Joiner, R. \& Traum, D. (1999) The role of grounding in collaborative learning tasks. In P. Dillenbourg (Ed) Collaborative learning: Cognitive and Computational Approaches (pp. 31-63) Oxford: Pergamon.

Brennan, S. E. (1991) Conversation with and through computers. User Modeling and User-Adapted Interaction, 1, pp. 67-86.

Bromme (2000) Beyond one's own perspective: The psychology of cognitive interdisciplinarity. In P. Weingart \& N. Stehr, (Eds), Practicing interdisciplinarity. (pp. 115-133)Toronto: Toronto University Press.

Canon-Bowers, J.A, Salas, E. \& Converse, S.A. (1993). Shared mental models in expert team decision making. In N.J. Castellan (Ed.). Individual and group decision making. (Pp. 221-246). Hillsdale, N.J: Lawrence Erlbaum.

Clark, H.H (1996), Using Language. Cambridge: Cambridge University Press.

Clark, H.H. \& Brennan S.E. (1991) Grounding in Communication. In L. Resnick, J. Levine \& S. Teasley (Eds.), Perspectives on Socially Shared Cognition (127-149). Hyattsville, MD: American Psychological Association.

Clark, H.H. \& Marshall, C.R (1981) Definite reference and mutual knowledge In A. K. Joshi, B. L. Webber, and I. A. Sag (Eds), Elements of Discourse Understanding. Cambridge University Press.

Clark, H.H. \& Wilkes-Gibbs, D (1986). Referring as a collaborative process. Cognition, 22:1-39.

Dillenbourg, P. (1999). What do you mean by collaborative learning? In P. Dillenbourg (Ed.), Collaborative learning: Cognitive and Computational Approaches (pp. 1-19). Oxford: Elsevier.

Dillenbourg, P. (2002). Over-scripting CSCL: The risks of blending collaborative learning with instructional design. In P. A. Kirschner (Ed). Three worlds of CSCL. Can we support CSCL (pp. 61-91). Heerlen, Open Universiteit Nederland.

Dillenbourg, P., Baker, M., Blaye, A., \& O'Malley, C. (1996) The evolution of research on collaborative learning. In E. Spada \& P. Reiman (Eds.), Learning in Humans and Machine: Towards an interdisciplinary learning science (pp. 189-211). Oxford: Elsevier.

Dillenbourg, P. \& Traum, P. (1999). Does a shared screen make a shared understanding ? C. Hoadley et J. Roschelle (eds), Proceedings of the Third Computer-Supported Collaborative Learning Conference, pp. 127-135, Stanford, Dec. 1999.

Dillenbourg, P., \& Traum, D. (2006). Sharing solutions: Persistence and grounding in multimodal collaborative problem solving. Journal of the Learning Sciences, 15 (1), 121-151.

Hutchins, E. (1995). How a cockpit remembers its speeds. Cognitive Science, 19, 265-288.

Kenny, D.A., Kashy, D. and Bolger, N. (1998): Data analysis in social psychology. In D. Gilbert, S. Fiske, \& G. Lindzey (eds.) Handbook of Social Psychology, vol. 1, Boston: McGraw-Hill, pp. 233-265.

Koschmann, T., \& LeBaron, C. (2003). Reconsidering common ground: Examining Clark’s contribution theory in the OR. In K. Kuutti, E. Karsten, G. Fitzpatrick, P. Dourish, \& K. Schmidt (Eds.), ECSCW 2003: Proc. EighthEuropean Conference on Computer-Supported Cooperative Work. Amsterdam: Kluwer.

Lockridge C.B. \& Brennan, S.E. (2002), Addressees' needs influence speakers' early syntactic choices, Psychonomic Bulletin \& Review, 9 (3), 550-557

Liben, L.S., Patterson, A.H, and Newcombe, N. (1981), Spatial Representation and Behavior Across the Life Span, New York: Academic Press.

Moreland RL. 2000. Transactive memory: learning who knows what in work groups and organizations. In Shared Cognition in Organizations: The Management of Knowledge, Thompson L, Messick D, Levine J (eds). Lawrence Erlbaum: Hillsdale, NJ; 3 \pm 31 .

Nova N., Wehrle, T., Goslin, J., Bourquin, Y. \& Dillenbourg, P. (2006): Collaboration in a Video Game : Impacts of Location Awareness. Journal of Multimedia, Tools and Applications.

Nova, N., Girardin, F. \& Dillenbourg, P. (2005): 'Location is not enough!’: an Empirical Study of LocationAwareness in Mobile Collaboration. Proceedings of the third IEEE International Workshop on Wireless and Mobile Technologies in Education, November 28-30, 2005, Tokushima, Japan, pp. 21-28, IEEE Press: Los Alamitos, California 
Paas, F., Renkl, A., \& Sweller, J. (2003). Cognitive load theory and instructional design: Recent developments. Educational Psychologist, 38, 1-4.

Roschelle, J. \& Teasley S.D. (1995) The construction of shared knowledge in collaborative problem solving. In C.E. O'Malley (Ed), Computer-Supported Collaborative Learning. (pp. 69-197). Berlin: Springer-Verlag

Sperber, D. \& D. Wilson (1986/1995) Relevance: Communication and Cognition. 2nd edition.

Suthers, D. D. (2006). Technology affordances for intersubjective meaning making: A research agenda for CSCL. To appear in International Journal of Computer-Supported Collaborative Learning (ijCSCL), 1(3).

Malle, Bertram F. (2003) Folk Theory of Mind: Conceptual Foundations of Social Cognition, in Hassin, Ran R. and Uleman, James S. and Bargh, John A., Eds. The new unconscious. Oxford University Press.

C., Greenberg, S., Roseman, R. (1996). Supporting Awareness of Others in Groupware. A short paper suite, in ACM SIGCHI'96 Conference on Human Factors in Computing System, Companion Proceedings, p205-215.

Pea, R. (1993) Practices of distributed intelligence and designs for education. In G. Salomon. (Ed). Distributed cognitions. Psychological and educational considerations (pp. 47-87) Cambridge, UK: Cambridge University Press.

Richardson, D.C \& Dale, R. (2005). Looking To Understand: The Coupling Between Speakers' and Listeners’ Eye Movements and its Relationship to Discourse Comprehension. Cognitive Science, 29, 1045-1060.

Salomon, G. (1993) No distribution without individual's cognition: a dynamic interactional view. In G. Salomon. (Ed). Distributed cognitions. Psychological and educational considerations (pp. 111-138) Cambridge, USA: Cambridge University Press.

Schober, M.F. (1993) Spatial perspective-taking in conversation. Cognition, 47, 1-24.

Schwartz, D.L. (1995). The emergence of abstract dyad representations in dyad problem solving. The Journal of the Learning Sciences, 4 (3), pp. 321-354.

Slugoski, B.R., Lalljee, M., Lamb, R. \& Ginsburg, G.P. (1993) Attribution in conversational context: Effect of mutual knowledge on explanation giving. European Journal of Social Psychology, 23 (219-238).

Smith, N. (1982) Mutual Knowledge, New York: Academic Press.

Wegner DM. 1987. Transactive memory: a contemporary analysis of the group mind. In Theories of Group Behavior, Mullen IB, Goethals GR (eds). Springer-Verlag: New York; $185 \pm 208$.

Wertsch, J.V. (1985) Adult-Child Interaction as a Source of Self-Regulation in Children. In S.R. Yussen (Ed).The growth of reflection in Children (pp. 69-97). Madison, Wisconsin: Academic Press.

\section{Acknowledgments}

We gratefully acknowledge the contribution of Fabien Girardin and Khaled Bachour. These researches were funded by the Swiss National Science Foundation (grant \#102511-106940). 